Sylvia de Oliveira e Cruz

Identificando Objetos Através de Pronome

Tese de Doutorado

Tese apresentada como requisito parcial para obtenção do título de Doutor pelo Programa de Pós-Graduação em Informática da PUC-Rio.

Orientador: Carlos José Pereira de Lucena

Volume I

Rio de Janeiro, agosto de 2003 


\title{
Identificando Objetos Através de Pronome
}

Tese apresentada como requisito parcial para obtenção do título de Doutor pelo Programa de Pós-Graduação em Informática da PUC-Rio. Aprovada pela Comissão Examinadora abaixo assinada.

\author{
Prof. Carlos José Pereira de Lucena \\ Orientador \\ Departamento de Informática - PUC-Rio \\ Prof. Roberto lerusalimschy \\ Departamento de Informática PUC-Rio
}

Prof. Noemi de La Rocque Rodriguez Departamento de Informática PUC-Rio

Maurício José Vianna e Silva Empresa MJV - Rio

Prof. Luiz Carlos Montez Monte Departamento de Ciência da Computação - UFF

Ney Dumont

Coordenador(a) Setorial do Centro Técnico Científico - PUC-Rio

Rio de Janeiro, 31 de março de 2003 
Todos os direitos reservados. É proibida a reprodução total ou parcial do trabalho sem autorização da universidade, da autora e do orientador.

\section{Sylvia de Oliveira e Cruz}

Graduou-se em Informática pela UFRJ (Universidade Federal do Rio de Janeiro) em 1988. Fez mestrado em Informática pela PUC-Rio em 1998 e doutorado, também em Informática pela PUC-Rio em 2003. Concentrou sua área de pesquisa em Engenharia de Software com ênfase em Arquitetura de Sistemas. Profissionalmente, sempre atuou no desenvolvimento de Sistemas. Participou do desenvolvimento do compilador da linguagem DIALOG (Clipper-like), do compilador da linguagem TOOL e da ferramenta CASE 2GOOD. Participou da elaboração a arquitetura do projeto MERCURIO da Marinha do Brasil e elaborou a arquitetura da solução de CRM da Vivo.

Ficha Catalográfica

Cruz, Sylvia de Oliveira e

Identificando objetos através de pronome / Sylvia de Oliveira e Cruz; orientador: Carlos José Pereira de Lucena. - Rio de Janeiro : PUC, Departamento de Informática, 2003.

125 f. ; il. ; $30 \mathrm{~cm}$

Tese (doutorado) - Pontifícia Universidade Católica do Rio de Janeiro, Departamento de Informática.

Inclui referências bibliográficas.

1. Informática - Teses. 2. Orientação a objetos. 3. Metaobjetos. 4. Linguagens abertas. 5. Framework. 6. Identificação de objetos. I. Lucena, Carlos J. P. de (Carlos José Pereira). II. Pontifícia Universidade Católica do Rio de Janeiro. Departamento de Informática. III. Título. 


\section{Agradecimentos}

Ao prof. Sérgio Carvalho (in memorian) pela honra de me ter confiado a exploração de idéias resultantes de toda uma vida dedicada a pesquisa em orientação a objetos.

Ao prof. Lucena por ter possibilitado que estas idéias fossem imortalizadas e por não ter me deixado esquecer que havia um objetivo a ser atingido.

Ao prof. Rangel (in memorian) por ter assumido a, ingrata mas fundamental, função de crítico deste trabalho quando seu maior crítico e principal interessado em sua qualidade faltou.

Ao prof. Alexandre Plastino pela ajuda na redação deste trabalho.

Aos companheiros de orientação pelo esforço em minimizar o vazio deixado pelo prof. Sérgio Carvalho.

À colega Christina von Flash, a Christina Baiana, pela alegria que sempre irradiou tornando menos árduas tarefas às vezes bem pouco animadas.

Ao funcionário público Alexandre Plastino por estar sempre pronto a me lembrar do compromisso assumido com o país ao iniciar este doutorado.

Ao Cnpq pelo apoio financeiro e pela confiança depositada.

Aos meus pais por sempre acreditarem em mim e por terem me ensinado o verdadeiro valor do saber.

A Lygia e a Branca por terem, por 5 anos, aceitado dividir a mãe com o computador.

Em especial, ao Alexandre por não ter se contentado com um papel único e por ter assumido, nestes 12 anos, a identidade de muito mais pronomes do que marido. 


\section{Resumo}

Cruz, Sylvia de Oliveira. Identificando Objetos Através de Pronome. Rio de Janeiro, 2003. 125p. Tese de Doutorado - Departamento de Informática, Pontifícia Universidade Católica do Rio de Janeiro.

A necessidade de se conhecer o identificador de um objeto para solicitar seus serviços é uma restrição à utilização de orientação a objetos na construção de sistemas grandes e complexos. Para minimizar esta restrição, foi proposta uma forma de se referenciar a um determinado objeto através de uma identificação genérica, denominada pronome. Pronomes permitem que o programador especifique o envio de uma mensagem sem que o objeto destino seja nominalmente conhecido neste contexto. Com pronomes, a visibilidade de um objeto aumenta sem que regras de encapsulamento e visibilidade sejam quebradas. Desta forma, classes servidoras completamente desacopladas de seus clientes podem ser construídas e utilizadas em diferentes contextos. Neste trabalho, exploram-se pronomes já definidos anteriormente, dando a estes um novo tratamento através de linguagens abertas, e definem-se novos pronomes. O conjunto de pronomes obtido facilita a implementação de arquiteturas e padrões de projeto de relevância, sendo também abrangente no que diz respeito às características dos objetos representados por seus pronomes. Define-se ainda, a partir da experiência adquirida com a implementação dos pronomes, um framework para a definição e implementação de novos pronomes. Este framework é ainda aqui instanciado para dois novos pronomes a fim de validá-lo.

\section{Palavras-chave}

Orientação a objetos; Metaobjetos; Linguagens Abertas; Framework; Identificação de objetos 


\section{Abstract}

Cruz, Sylvia de Oliveira. Identifying Objects Through Pronouns. Rio de Janeiro, 2003. 125p. PhD Thesis - Departamento de Informática, Pontifícia Universidade Católica do Rio de Janeiro.

The need to know the object identification to request it's services is a restriction in the construction of object oriented system. To reduce this restriction we have proposed a form of generic reference to an object named pronoum. Pronouns allow the programmer to specify how to send a message without knowing the destination object by name. With pronouns, the visibility of an object increases without breaking the rule of encapsulation. With this feature decoupled server classes may be constructed and used in different contexts. This work define some pronouns, show its utilities through of examples and suggest a framework to new pronouns creation.

\section{Keywords}

Object Oriented; Metaobjects; Open Languages; Framework; Objects identification 


\section{Sumário}

1 Introdução

2 Comunicação Baseada em Pronomes 19

$\begin{array}{lll}2.1 & \text { Apresentação } & 19\end{array}$

2.2 Semântica de distribuição de mensagens através de pronomes 21

2.3 Semântica de recebimento de mensagens através de pronomes 22

2.4 Aplicações 23

2.4.1 Distributed Callback [MM97] 23

2.4.2 Algoritmo Paralelo de Divisão e Conquista 25

2.4.3 Mediator $[\mathrm{G}+95] \quad 28$

2.4.4 Invocação Implícita [SN92] 29

$\begin{array}{lll}2.4 .5 & 31\end{array}$

2.4.6 Singleton $[\mathrm{G}+95]$

2.5 Linguagens Abertas 34

2.6 Trabalhos Relacionados 38

3 O Pronome SENDER $\quad 41$

3.1 O Objeto Emissor da Mensagem Corrente 41

3.2 Transformações de Código 42

3.3 O Nível Meta 44

3.4 Distributed Callback utilizando SENDER 47

4 O Pronome CREATOR $\quad 51$

4.1 O objeto criador do objeto corrente 51

4.2 Transformações de Código 52

4.3 O Nível Meta 54

4.4 O Algoritmo de Divisão e Conquista Utilizando CREATOR 57

5 O Pronome PARENT $\quad 61$

5.1 O Objeto que Declarou o Objeto Corrente da Execução 61 
5.2 Transformações de Código 62

5.3 O Nível Meta 64

$\begin{array}{lll}5.4 & \text { O Padrão Mediator Utilizando PARENT }\end{array}$

6 O Pronome MAIN $\quad 70$

6.1 O Objeto Principal do Sistema 70

$\begin{array}{lll}6.2 & \text { Transformações de Código } & 71\end{array}$

6.3 O Nível Meta 72

6.4 O Padrão Singleton Utilizando MAIN 75

7 O Pronome ALL $\quad 78$

$\begin{array}{lll}7.1 & \text { O Conjunto de Todos os Objetos do Sistema }\end{array}$

$\begin{array}{lll}7.2 & \text { Transformações de Código } & 79\end{array}$

$\begin{array}{lll}7.3 & \text { O Nível Meta } & 80\end{array}$

7.4 Invocação Implícita Utilizando ALL 82

8 O Pronome ANY $\quad 85$

8.1 Um Objeto Qualquer $\quad 85$

8.2 Transformações de Código 86

$\begin{array}{lll}8.3 & \text { O Nível Meta } & 88\end{array}$

8.4 Padrão Arquitetural Broker Utilizando ALL 89

9 Definindo Novos Pronomes 93

9.1 A Abstração do Framework 93

9.2 O Framework 94

9.2.1 Primeiro Nível de Abstração 96

9.2.2 Categorização de Pronomes 97

9.2.3 Subconjunto de Objetos do Sistema 100

9.2.4 Categorização das relações com o objeto corrente da execução 102

9.2.5 Categorização das Relações Estruturais 104

9.3 Instanciação do Framework para a Obtenção do Pronome MAIN 106

9.4 Instanciação do Framework para a Obtenção do Pronome ALL 108

9.5 Instanciação do Framework para a Obtenção do Pronome ANY 109

9.6 Instanciação do Framework para a Obtenção do Pronome SENDER 110

9.7 Instanciação do Framework para a Obtenção do Pronome CREATOR 111

9.8 Instanciação do Framework para a Obtenção do Pronome PARENT 112

9.9 A Instanciação do Framework para a Obtenção de Novos Pronomes 113 
$\begin{array}{lll}\text { 9.9.1 O Pronome CLOCK } & 114\end{array}$

9.9.2 A Obtenção do Pronome CLOCK a Partir do Framework 115

$\begin{array}{lll}\text { 9.9.3 O Pronome SIBLINGS } & 116\end{array}$

10 Conclusão 119

11 Referências bibliográficas 122 


\section{Lista de Figuras}

Figura 2.1 - Solução do Padrão Distributed Callback..................................... 24

Figura 2.2 - Solução para o problema da quadratura utilizando bolsa de tarefas 27

Figura 2.3 - Estrutura do padrão Mediator . 29

Figura 2.4 - Comportamento do padrão Broker atendendo a uma requisição de um cliente 31

Figura 2.5 - Estrutura do padrão Singleton 33

Figura 2.6 - Esquema de obtenção do código executável em linguagens abertas 37

Figura 3.1 - Cenário no nível base de utilização do pronome SENDER ............. 42

Figura 3.2 - Cenário de utilização do pronome SENDER transformado ............. 44

Figura 3.3 - Nível Meta para implementação do pronome SENDER ................. 45

Figura 3.4 - Repositório de mensagens disponibilizado como suporte de tempo de execução 46

Figura 3.5 - Esquema da implementação do padrão Distributed Callback utilizando SENDER 47

Figura 3.6 - Gráfico de comparação entre os tempos de execução do padrão Distributed Callback. 49

Figura 4.1 - Cenário de utilização do pronome CREATOR em nível base ........ 52

Figura 4.2 - Cenário de utilização do pronome CREATOR transformado .......... 53

Figura 4.3 - Nível Meta para implementação do pronome CREATOR ............... 55

Figura 4.4 - Esquema da implementação do algoritmo paralelo de divisão e conquista utilizando CREATOR 57

Figura 4.5 - Gráfico de comparação entre os tempos de execução do algoritmo

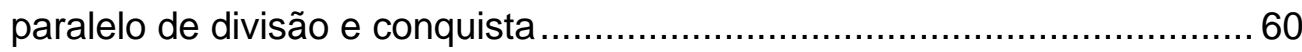

Figura 5.1 - Cenário de utilização do pronome PARENT em nível base ........... 62

Figura 5.2 - Cenário de utilização do pronome PARENT transformado ............. 64

Figura 5.3 - Nível Meta para implementação do pronome PARENT ..................6. 65

Figura 5.4 - Esquema da implementação do padrão Mediator utilizando PARENT 
Figura 5.5 - Gráfico de comparação entre os tempos de execução do padrão

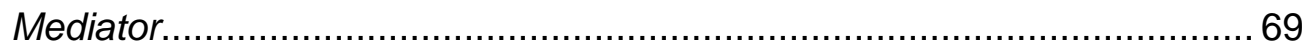

Figura 6.1 - Cenário de utilização do pronome MAIN em nível base................. 71

Figura 6.2 - Cenário de utilização do pronome MAIN transformado .................. 72

Figura 6.3 - Nível Meta para implementação do pronome MAIN ....................... 73

Figura 6.4 - Esquema da implementação do padrão Singleton utilizando MAIN 75

Figura 6.5 - Gráfico de comparação entre os tempos de execução do padrão

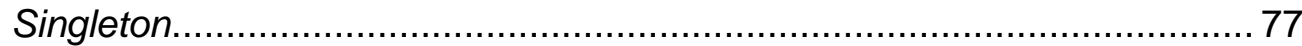

Figura 7.1 - Cenário de utilização do pronome ALL em nível base ................... 79

Figura 7.2 - Cenário de utilização do pronome ALL transformado ..................... 80

Figura 7.3 - Nível Meta para implementação do pronome ALL ......................... 81

Figura 7.4 - Gráfico de comparação entre os tempos de execução da Invocação

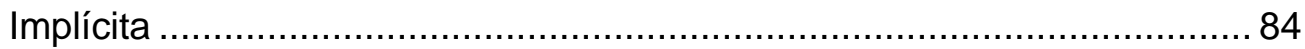

Figura 8.1 - Cenário de utilização do pronome ANY em nível base .................. 86

Figura 8.2 - Cenário de utilização do pronome ANY transformado ................... 87

Figura 8.3 - Suporte de tempo de execução para utilização do pronome ANY . 88

Figura 8.4 - Nível Meta para implementação do pronome ANY ........................ 88

Figura 8.5 - Gráfico de comparação entre os tempos de execução do padrão

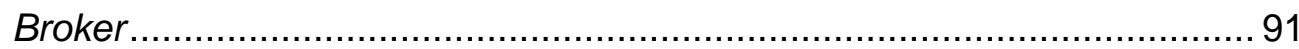

Figura 9.1 -Framework para suporte de tempo de execução ........................... 95

Figura 9.2 - Nível Meta do framework .......................................................... 95

Figura 9.3 - Primeiro nível de abstração do framework .................................. 96

Figura 9.4 - Categorização dos pronomes segundo suas características de

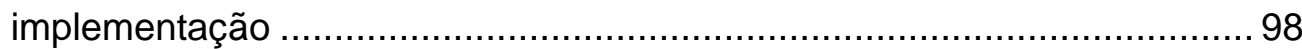

Figura 9.5 - Subconjuntos de objetos do sistema....................................... 101

Figura 9.6 - Categorização de pronomes relacionados com o objeto corrente da

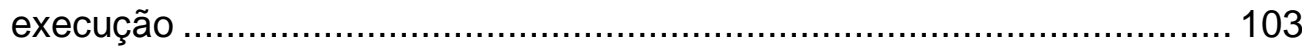

Figura 9.7 - Categorização dos pronomes que representam objetos relacionados estruturalmente com o objeto corrente da execução............................... 104

Figura 9.8 - Implementação do pronome MAIN através do framework........... 107

Figura 9.9 - Implementação do pronome ALL através do framework ............... 108

Figura 9.10 - Implementação do pronome ANY através do framework ........... 109

Figura 9.11 - Implementação do pronome SENDER através do framework.... 110

Figura 9.12 - Implementação do pronome CREATOR através do framework. 111

Figura 9.13 - Implementação do pronome PARENT através do framework ....112

Figura 9.14 - Processos executando ações locais e de comunicação.............. 114

Figura 9.15 - Implementação do pronome CLOCK através do framework ......115 
Figura 9.16 - Implementação do pronome SIBLINGS através do framework .. 117 


\section{Lista de Tabelas}

Tabela 3.1 - Tempos de execução do padrão Distributed Callback................... 48

Tabela 3.2 - Desvios Padrões das amostras de tempo utilizadas para análise da execução do padrão Distributed Callback. 49

Tabela 4.1 - Tempos de execução do algoritmo paralelo de divisão e conquista

59

Tabela 4.2 - Desvios Padrões das amostras de tempo utilizadas para análise da execução do algoritmo paralelo de divisão e conquista ............................ 59

Tabela 5.1 - Tempos de execução do padrão Mediator ................................... 68

Tabela 5.2 - Desvios Padrões das amostras de tempo utilizadas para análise da execução do padrão Mediator.............................................................. 68

Tabela 6.1 - Tempos de execução do padrão Singleton ................................... 76

Tabela 6.2 - Desvios Padrões das amostras de tempo utilizadas para análise da execução do padrão Singleton............................................................ 76

Tabela 7.1 - Tempos de execução da Invocação Implícita.................................8 83

Tabela 7.2 - Desvios Padrões das amostras de tempo utilizadas para análise da

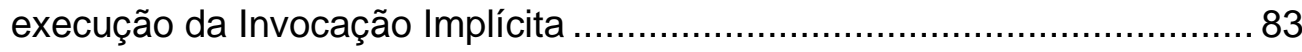

Tabela 8.1 - Tempos de execução do padrão estrutural Broker....................... 90

Tabela 8.2 - Desvios Padrões das amostras de tempo utilizadas para análise do padrão estrutural Broker ..................................................................... 91 